\title{
Magnetic field effect on the sheath thickness in plasma immersion ion implantation
}

\author{
M. Keidara) \\ Department of Aerospace Engineering, The University of Michigan, Ann Arbor, Michigan 48109 \\ O. R. Monteiro and A. Anders \\ Lawrence Berkeley National Laboratory, MS 53, University of California, Berkeley, California 94720 \\ I. D. Boyd \\ Department of Aerospace Engineering, The University of Michigan, Ann Arbor, Michigan 48109
}

(Received 22 April 2002; accepted for publication 17 June 2002)

\begin{abstract}
The sheath thickness in plasma immersion ion implantation has been investigated in the presence of a transverse magnetic field. It has been found that the steady-state sheath thickness increases with increasing magnetic field strength. This result is in line with a simplified model of the sheath in which the steady-state sheath thickness is determined by the plasma density and ion velocity at the sheath edge. These results suggest that a magnetic field may be used to control the high-voltage sheath in plasma immersion ion implantation. (C) 2002 American Institute of Physics.
\end{abstract}

[DOI: 10.1063/1.1499516]

Plasma immersion ion implantation (PIII) is a relatively new technology developed in the 1980's. ${ }^{1,2}$ PIII is used to modify material surface properties and is an alternative approach to beamline ion implantation. In a PIII process, a negatively biased target is placed in plasma. The resulting electric field is concentrated in the target vicinity, the sheath, i.e., a region where quasineutrality breaks down. This electric field accelerates ions to high energy towards the target. The high energy ions are implanted and thereby the surface properties can be modified for a wide range of materials. Since the main ion acceleration is concentrated in the sheath, sheath behavior is extremely important in PIII-related processes.

In the simplest PIII case, the target is immersed into a gaseous, stationary plasma. Such a configuration has benefits such as the ability of treating targets with complex geometry. A related kind of surface modification has been developed based on the use of vacuum arc generated metal plasma. 3,4 This technique incorporates both surface deposition of plasma and subsurface ion implantation, with energetic ion implantation occurring during the voltage-on part of the substrate pulse-biasing and deposition occurring during the voltage-off part of the bias cycle. It was shown that in this process film adhesion is improved in comparison with pure deposition. Peculiarities of such PIII variants were sufficiently described in recent reviews ${ }^{2,5,6}$ and sheath measurements were reported in Ref. 7.

Application of negative bias to the substrate immersed in the plasma leads to sheath formation near the substrate. An ion matrix sheath is established on a time scale of the inverse electron plasma frequency, and ions respond much slower, namely on a time scale of the inverse ion plasma frequency. During this time the high voltage sheath edge propagates into the plasma at about the ion acoustic speed. ${ }^{1,8}$ A growing sheath may deplete the plasma and may stop the process when the sheath expands all the way to the chamber wall, however, this will only occur at relatively low plasma densi-

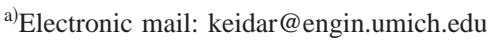

ties and high bias voltages. At higher plasma densities, the sheath will reach a steady-state position.

Using a vacuum arc plasma source for PIII leads to several effects. The ions in the vacuum arc plasma jet have supersonic velocity (gained in the cathode spot region), which can significantly affect sheath expansion. ${ }^{9}$ The plasma density along the plasma jet is nonuniform, which may lead to a plasma density increase at the sheath edge during sheath expansion. ${ }^{10}$ In addition, ion current increases with increasing bias voltage, which was also explained in terms of plasma nonuniformity. ${ }^{11}$ Plasma drift generally leads to a smaller sheath thickness. If the sheath is too thin there is a problem associated with electrical breakdown due to very high electric field strength at the target surface. ${ }^{12}$ Sheath optimization issues were considered recently. ${ }^{13}$ It is clear that keeping the sheath thickness in the optimal range is an important issue.

One of the ways to control sheath thickness may be the use of a magnetic field. It is well known that a sheath is significantly affected by a magnetic field, especially in the case when the magnetic field is parallel to the surface or intersects the surface at small angles. ${ }^{14}$ Therefore, it is expected that a magnetic field will affect the sheath dynamics under PIII conditions as well. Previously, magnetic fields have been considered in PIII to suppress secondary electrons and the associated generation of $\mathrm{x}$-rays. ${ }^{2,15}$ Here we focus on measurements of the sheath thickness rather than secondary electrons.

In this letter we present some preliminary results of experimental measurements of the sheath size using a filtered vacuum arc plasma source. A simplified schematic of the experimental setup is shown in Fig. 1. This setup is similar to that described in Ref. 7. A vacuum arc plasma gun with a silver cathode was operated with an arc current of $500 \mathrm{~A}$ in pulses of $250 \mu$ s duration and with a repetition rate of one pulse per second. The negative bias pulse was of magnitude up to $-8 \mathrm{kV}$. The plasma was injected into a $90^{\circ}$ curved magnetic duct in order to separate out macroparticles (cathode debris). The spherical target was located downstream of 


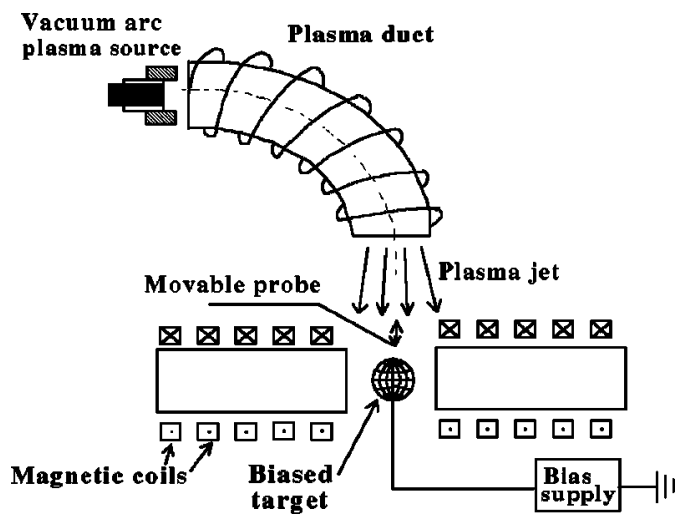

FIG. 1. Schematic of the experimental configuration (not to scale).

the duct at $25 \mathrm{~cm}$ from the duct exit plane. The voltage was applied using a high-voltage pulse modulator. The voltage and magnetic field were applied with a delay with respect to the arc pulse. An example of arc pulse, magnetic field and bias voltage ( $1 \mathrm{kV}$ case) profile is shown in Fig. 2(a). The magnetic field was applied using two solenoids of $22.9 \mathrm{~cm}$ length and $9 \mathrm{~cm}$ diameter with 48 turns each connected in series. The two coils were separated by $9 \mathrm{~cm}$ and the target was placed between them as shown in Fig. 1. The magnetic field at the edge of a solenoid can be calculated as: $B$ $=\mu n I / 2$, where $I$ is the current in the coils and $n$ is the number of turns per length. In the following, we will present results as a function of this reference magnetic field. The target shown in Fig. 1 is a biased aluminum sphere of 2.54 $\mathrm{cm}$ diameter supported by a ceramic holder. The magnetic field has its main component parallel to the surface area facing the plasma flow (transverse field).

A small, positively biased $(75 \mathrm{~V})$, movable cylindrical probe of $4.5 \mathrm{~mm}$ length and $2.2 \mathrm{~mm}$ diameter was used to determine the size of the sheath as a function of magnetic field and bias voltage. When the probe was immersed in the plasma, it collected the electron saturation current and when the probe was immersed in the sheath, its current is practically zero because the sheath is depleted of electrons. By moving the probe from the target, it was possible to determine the sheath edge distance from the target when observing a sharp change in electron current collected by the probe. The same measuring principle was previously used for measurements of expanding sheaths with low-density, stationary gaseous plasma ${ }^{16}$ and in vacuum arc plasmas. ${ }^{7}$ The bias voltage was varied in order to find the voltage at which electron current to the probe will be zero for a fixed probe location. In doing the measurements this way we eliminated the error associated with probe positioning. We define the "critical" bias voltage $U_{\text {cr }}$ as the minimum target voltage at which the current to the probe becomes zero. The bias voltage $U_{\text {cr }}$ corresponds to the case when the sheath thickness equals the distance of the probe to the target.

Figure 2(b) shows the electron current measured with the probe as a function of the bias voltage for the fixed probe position and magnetic field. The probe shows a large electron current signal when target bias voltage is small. The noise is associated with the plasma generation process. With increasing bias voltage, the probe signal decreases and suddenly becomes very small as sheath thickness reaches the probe position. When the probe is located within the electrondepleted sheath, the probe current is very small. We found
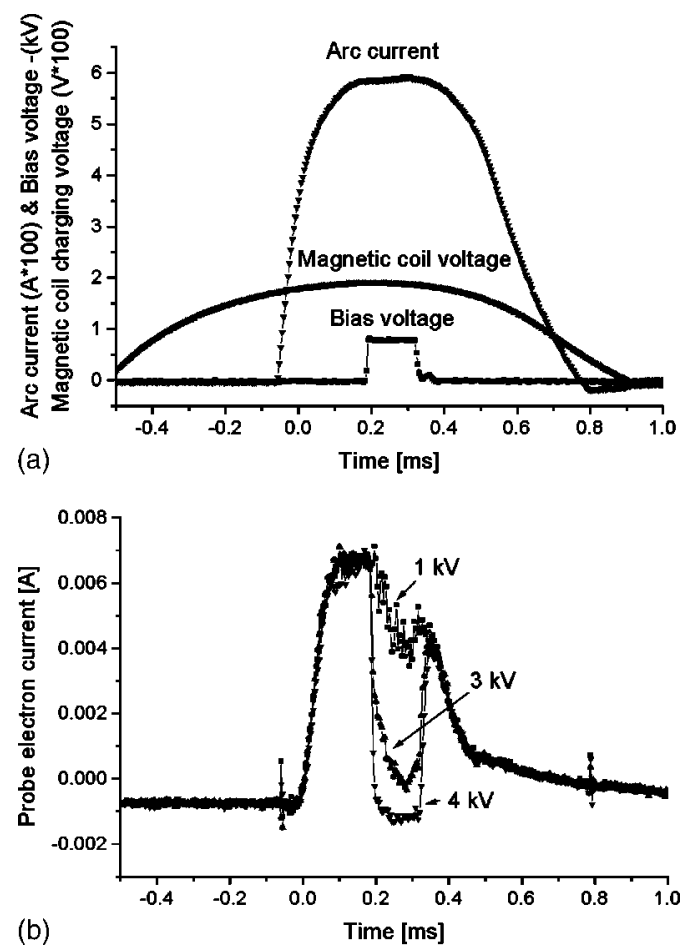

FIG. 2. (a) Arc current, magnetic field, target voltage. (b) Electron saturation current of a probe with bias voltage as a parameter. Probe is located at $2 \mathrm{~mm}$ from the target and magnetic field is $0.023 \mathrm{~T}$.

that the critical voltage $U_{\text {cr }}$ generally decreases with increasing magnetic field. For instance, investigating a distance of 2 $\mathrm{mm}$ from the target surface, the critical voltage decreases from $6 \mathrm{kV}$ in the case of zero magnetic field to $3 \mathrm{kV}$ in the case of $0.1 \mathrm{~T}$ magnetic field.

The influence of the magnetic field effect can be viewed such that the sheath thickness increases with magnetic field for a fixed bias voltage. The behavior of the critical voltage as a function of the magnetic field for several probe locations can be used to derive a sheath thickness plot as a function of the applied bias, with the magnetic field as a parameter. We included the probe diameter in our definition of the sheath thickness. The sheath thickness dependence on the bias voltage is shown in Fig. 3 with magnetic field as a parameter. As anticipated, the results confirm that the magnetic field has a significant effect on the high voltage sheath development.

In the following we propose a qualitative explanation for the observed phenomena. Our explanation is in line with the basic model of plasma immersion ion implantation as formulated by Lieberman. ${ }^{8}$ When a negative voltage is applied to a substrate immersed in a plasma, electrons are repelled from the substrate, leading to sheath formation. Electrons drift across the magnetic field away from the target due to the presence of the high electric field and rare collisions with ions and neutrals. Rare collisions will lead to a depletion of the sheath from electrons and therefore conditions close to pure space charge sheath are realized. The ions are then accelerated toward the substrate by the electric field of the sheath. In the one-dimensional steady state case the sheath thickness can be estimated according to the Child-Langmuir law $^{17,18}$

$$
s=\left(\frac{4}{9} \varepsilon\right)^{1 / 2}\left(\frac{2 Z_{i} e}{m_{i}}\right)^{1 / 4} \frac{U^{3 / 4}}{\left(e Z_{i} N V\right)^{1 / 2}},
$$

where $V$ is the ion velocity at the sheath edge, $U$ is the 


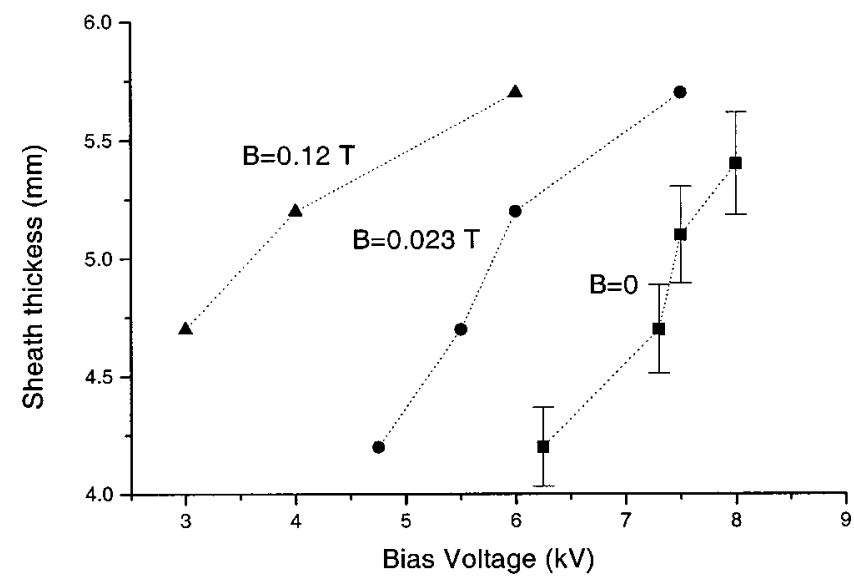

FIG. 3. Sheath thickness plot as a function of the applied bias, with the magnetic field as a parameter.

voltage across the sheath, $s$ is the sheath thickness, $\varepsilon$ is the permittivity of vacuum, $Z_{i}$ is the ion mean charge number, $N$ is the plasma density at the sheath edge, and $m_{i}$ is the ion mass. In this equation plasma density and velocity at the sheath edge determine the sheath thickness for given bias voltage. Strictly speaking, Eq. (1) can be questioned since in the vacuum are plasma ions are supersonic (for instance in the case of the silver ion velocity is about $\left.1.0 \times 10^{4} \mathrm{~m} / \mathrm{s}\right),{ }^{19}$ while Eq. (1) corresponds to the case of zero ion velocity at the sheath edge. However, as it was discussed in Ref. 7, in the case of the high voltage sheath this equation can be still acceptable since $U / E_{0} \gg 1$ where $E_{0}$ is the energy of ions at the sheath edge. One can see that the steady-state sheath thickness is determined by plasma density and ion velocity at the sheath edge for a given bias voltage. While plasma density and velocity distribution in the vacuum arc plasma jet are well known, ${ }^{20,21}$ plasma density and velocity at the sheath edge in the specific configuration of our experiment are determined by the plasma jet dynamics across magnetic field layer in the target vicinity.

The problem of the plasma propagation across magnetic field is a long standing one having numerous applications. ${ }^{22-24}$ In a simple quasione-dimensional approximation the potential distribution across the magnetic layer can be calculated as

$$
\frac{d \varphi}{d x}=\frac{k T_{e}}{e} \frac{d \ln N}{d x}+\beta B V-\frac{j}{\sigma},
$$

where $x$ is the direction along plasma jet normal to the target, $\varphi$ is the plasma potential, $\beta$ is the Hall parameter, $B$ is the magnetic field, $j$ is the current density, $T_{e}$ is the electron temperature, and $\sigma$ is the conductivity. Comparison of the two last terms on the right hand of Eq. (2) shows that the last term in Eq. (2) is negligible if $B>10^{-20} N$. In our experiment we have measured a plasma density of about $10^{17} \mathrm{~m}^{-3}$ in the target vicinity. Therefore, the conclusion of Ref. 24 , where a currentless magnetic layer was considered, can be applied here in the entire range of magnetic fields. This means that when a partially magnetized plasma (i.e., only electrons are magnetized) flows across a magnetic field, the plasma potential increases. This potential behavior provides quasineutrality across the layer. As a result, the ion velocity decreases as plasma propagates across the layer. In the quasione-dimensional approximation (i.e., assuming that the plasma expands radially with constant velocity), plasma jet spreading in the radial direction can be calculated as $d r / d x$ $=C_{s} / V$, where $C_{s}$ is the sound speed. Taking this into account one can conclude that when the axial plasma velocity decreases (as a result of the magnetic field effect) it leads to plasma spreading in the radial direction (i.e. along magnetic field lines) followed by plasma density decreases. Thus plasma jet propagation across a magnetic field layer leads to decrease in the plasma density and ion velocity in the quasi one-dimensional approximation. A similar effect was considered in Ref. 24. This means that the plasma density and ion velocity at the sheath edge decrease with magnetic field and according to Eq. (1), the steady state sheath thickness increases. This conclusion is in line with our experimental observations.

In summary, we have found that a transverse magnetic field affects the high voltage sheath dynamics. We found that a critical voltage (defined as the voltage at which the electron current to the probe becomes zero at a given probe location from the target) generally decreases with magnetic field. This means that the sheath thickness increases with magnetic field for a fixed bias voltage. This result can be explained in terms of a simple model of the sheath, when the steady-state sheath thickness is determined by the plasma density and ion velocity at the sheath edge. These results show that a magnetic field may be used to control high-voltage sheath dynamics.

The authors thank Ian Brown for valuable discussions and support and Tom Oates for assistance with experimental measurements. This work was supported in part by the U.S. Department of Energy, under Contract No. DE-AC0376 SF00098.

${ }^{1}$ J. R. Conrad, J. L. Radtke, R. A. Dodd, F. J. Worzala, and N. C. Tran, J. Appl. Phys. 62, 4591 (1987).

${ }^{2}$ Handbook of Plasma Immersion Ion Implantation and Deposition, edited by A. Anders (Wiley, New York, 2000).

${ }^{3}$ I. G. Brown, X. Godechot, and K. M. Yu, Appl. Phys. Lett. 58, 1392 (1991)

${ }^{4}$ I. G. Brown, A. Anders, S. Anders, M. R. Dickinson, I. C. Ivanov, R. A. MacGill, X. Yao, and K. M. Yu, Nucl. Instrum. Methods Phys. Res. B 80/81, 1281 (1993).

${ }^{5}$ A. Anders, Surf. Coat. Technol. 93, 157 (1997).

${ }^{6}$ I. G. Brown, Surf. Coat. Technol. 136, 16 (2000).

${ }^{7}$ A. Anders, Surf. Coat. Technol. 136, 85 (2001).

${ }^{8}$ M. A. Lieberman, J. Appl. Phys. 66, 2926 (1989).

${ }^{9}$ I. G. Brown, O. Monteiro, and M. M. M. Bilek, Appl. Phys. Lett. 74, 2426 (1999).

${ }^{10}$ M. Keidar and I. G. Brown, J. Vac. Sci. Technol. B 17, 2648 (1999).

${ }^{11}$ M. Keidar, O. Monteiro, and I. G. Brown, Appl. Phys. Lett. 76, 3002 (2000).

${ }^{12}$ A. Anders, Appl. Phys. Lett. 76, 28 (2000).

${ }^{13}$ M. M. M. Bilek, J. Appl. Phys. 89, 923 (2001).

${ }^{14}$ M. Keidar and I. I. Beilis, Appl. Phys. Lett. 73, 306 (1998).

${ }^{15}$ D. J. Rej, B. P. Wood, R. J. Faehl, and H. H. Fleischmann, J. Vac. Sci. Technol. B 12, 861 (1994).

${ }^{16}$ M. Shamim, J. T. Scheuer, and J. R. Conrad, J. Appl. Phys. 69, 2904 (1991).

${ }^{17}$ C. D. Child, Phys. Rev. 32, 492 (1911)

${ }^{18}$ I. Langmuir, Phys. Rev. 2, 450 (1913).

${ }^{19}$ G. Yushkov, A. Anders, E. M. Oks, and I. G. Brown, J. Appl. Phys. 88, 5618 (2000)

${ }^{20}$ B. Jüttner, IEEE Trans. Plasma Sci. PS-15, 474 (1987).

${ }^{21}$ M. Keidar, I. Beilis, R. L. Boxman, and S. Goldsmith, J. Phys. D 29, 1973 (1996).

${ }^{22}$ D. A. Baker and J. E. Hammer, Phys. Rev. Lett. 8, 157 (1962).

${ }^{23}$ O. Buneman, IEEE Trans. Plasma Sci. 20, 672 (1992).

${ }^{24}$ M. Keidar and I. D. Boyd, J. Appl. Phys. 86, 4786 (1999). 